\title{
1 Towards an Ecosystem Model of Infectious Disease
}

3 James M. Hassell ${ }^{1,2^{*}}$, Tim Newbold ${ }^{3}$, Andrew P. Dobson ${ }^{4}$, Yvonne Linton ${ }^{5,6,7}$, Lydia H.V.

4 Franklinos $^{3}$, Dawn Zimmerman ${ }^{1,2}$, Katrina M. Pagenkopp Lohan ${ }^{8}$

$6{ }^{1}$ Global Health Program, Smithsonian Conservation Biology Institute, Washington DC, USA

$7 \quad{ }^{2}$ Department of Epidemiology of Microbial Disease, Yale School of Public Health, New Haven, 8 CT, USA

$9{ }^{3}$ Centre for Biodiversity \& Environment Research (CBER), Department of Genetics, Evolution

10 and Environment, University College London, London, UK

$11{ }^{4}$ Department of Ecology \& Evolutionary Biology, Princeton University, Princeton, NJ, USA

$12{ }^{5}$ Walter Reed Biosystematics Unit (WRBU), Smithsonian Institution Museum Support Center,

13 Suitland, MD, USA

$14{ }^{6}$ Department of Entomology, Smithsonian National Museum of Natural History, Washington,

15 DC, USA

$16{ }^{7}$ Walter Reed Army Institute of Research (WRAIR), 503 Robert Grant Ave, Silver Spring MD

17 20910, USA.

$18{ }^{8}$ Marine Disease Ecology Laboratory, Smithsonian Environmental Research Center, Edgewater, 19 MD, USA

$21 *$ Corresponding author 


\section{Abstract}

25 Increasingly intimate associations between human society and the natural environment are

26 driving the emergence of novel pathogens, with devastating consequences for humans and

27 animals alike. Prior to emergence, these pathogens exist within complex ecological systems that

28 are characterized by trophic interactions between parasites, their hosts, and the environment.

29 Predicting how disturbance to these ecological systems places people and animals at risk from

30 emerging pathogens - and the best ways to manage this - remains a significant challenge.

31 Predictive systems ecology models are powerful tools for the reconstruction of ecosystem

32 function but have yet to be considered for modeling infectious disease. Part of this stems from a

33 mistaken tendency to forget about the role that pathogens play in structuring the abundance and

34 interactions of the free-living species favored by systems ecologists. Here, we explore how

35 developing and applying these more complete systems ecology models at a landscape scale

36 would greatly enhance our understanding of the reciprocal interactions between parasites,

37 pathogens and the environment, placing zoonoses in an ecological context, while identifying key

38 variables and simplifying assumptions that underly pathogen host switching and animal-to-

39 human spillover risk. As well as transforming our understanding of disease ecology, this would

40 also allow us to better direct resources in preparation for future pandemics.

\section{Introduction}

43 Emerging infectious diseases (EIDs) are increasing in frequency as global environmental and

44 anthropogenic changes accelerate ${ }^{1-3}$. For animal-to-human (zoonotic) spillover and subsequent

45 pathogen amplification to occur, a complex set of epidemiological, ecological and behavioral

46 conditions that influence the composition, infection dynamics, contact rates and likelihood of 
47 infection within and between host populations must align ${ }^{4}$. Mitigation of future pandemics will

48 rely on our ability to understand how these mechanisms converge to result in exposure of people

49 to novel pathogens, and identify areas at higher risk of pathogen spillover, so that limited

50 resources for animal and human surveillance and risk mitigation efforts can be proactively

51 directed to these sites ${ }^{5}$.

52 Accurate forecasting of spillover risk requires a clear understanding of the pathogen

53 dynamics at play in differing global biomes. Interactions between parasites (throughout this

54 article we use the term parasite to describe all pathogenic (disease causing) and non-pathogenic

55 organisms that colonize and can be transmitted between hosts), their hosts, vectors and the

56 environment over defined geographic and temporal scales can be thought of as "episystems",

57 (Figure 1). Pathogen communities are focal points of episystems, where competition and co-

58 existence between pathogens and commensal organisms for resources within hosts regulates

59 virulence and transmission, while exerting effects on host fitness and behavior that percolate

60 across trophic scales. The composition and function of these parasite communities are also

61 defined by the top-down impacts of environmental conditions on the fitness, distribution and

62 interactions between host populations. By linking host population dynamics to the composition

63 and turnover of parasite communities inhabiting these host 'patches', metacommunity theory can

64 be used to place zoonotic pathogens and their emergence into new host populations in an

65 ecological context (an approach we refer to as 'pathogen community ecology') ${ }^{8,9}$. While

66 empirical investigations can reveal important associations between host and parasite

67 communities (e.g. ${ }^{10-13}$ ), modeling of the fundamental processes underpinning these relationships

68 provides the only replicable opportunity to understand how natural and human-driven changes to

69 these systems modify the risks that pathogens pose to humans, and to forecast change in these 
70 risks. The scale of this computationally intensive task — compounded by limited data, complex

71 and often nonlinear relationships, and high levels of uncertainty — has so far eluded conventional

72 epidemiological approaches. We propose that rescaling and novel structural reorganization of

73 models for these systems now make this goal attainable.

74 Our understanding of infectious disease transmission has come a long way in the past 30

75 years ${ }^{14,15}$; modern epidemiological models facilitate more accurate predictions about pathogen

76 transmission and disease risk than ever before. Being rooted within foundational concepts of

77 single-agent, single-host systems (such as the basic reproductive number R0), most existing

78 epidemiological models - including more recent frameworks such as stochastic metacommunity

79 models and multi-pathogen SIR models - require significant modifications if they are required to

80 explore the interactions and feedback loops that exist between multiple pathogens, hosts and

81 their shared environment ${ }^{8,16,17}$. Statistical and machine learning methods that have been adapted

82 from ecology (e.g. species distribution models, hierarchical spatio-temporal models, joint species

83 distribution models) have made significant contributions to public health by mapping infectious

84 disease risk and are capable of identifying relationships between zoonotic pathogens, parasite

85 communities, macro fauna and ecosystem structure and function ${ }^{18-20}$. However, using these top-

86 down approaches to extrapolate beyond existing conditions can be problematic, as they lack a

87 mechanistic framework with which to test the impact of management changes and interventions

88 on infectious diseases ${ }^{21-23}$.

89 Whole systems approaches, akin to those used to forecast the world's weather, study

90 biological regulation within the human body, and manage the World's fisheries, are increasingly

91 applied in ecology to understand how anthropogenic forces (such as climate change) change the

92 behavior of ecological systems. Predictive systems ecology ${ }^{24}$ promotes the use of mechanistic, 
93 process-based models, parameterized by observational and experimental data, to understand and

94 predict the future state of ecological systems. Outputs are 'emergent properties' of these models

95 - quantitative measures for how different components of the ecosystem change over time.

96 Models of terrestrial and ocean ecosystems (e.g. dynamic global vegetation models, ocean

97 ecosystem models, general ecosystem models $)^{25}$ have been used to generate estimates of primary

98 production from forests, community structure of phytoplankton, and have recently been extended

99 to model the World's ecosystems ${ }^{26}$. Unfortunately, none of these approaches consider hosts and

100 their parasites, which exert a ubiquitous influence on all free-living species. We believe that now

101 is the time to extend this approach into the fields of epidemiology and disease ecology ${ }^{27}$.

102 Applying systems-level thinking to forecast disease emergence will necessitate a

103 fundamental change in how we conceptualize infectious diseases. In much the same way that a

104 mechanic working to improve the future performance of a race car requires complete knowledge

105 of how its engineered components are assembled and interact during operation, practitioners

106 looking to predict and affect the future state of episystems require models that capture the suite

107 of biological and social mechanisms underpinning the behavior of host and pathogen

108 communities. Process-based models, in which the fundamental ecological and epidemiological

109 mechanisms determining disease risk are described in a mathematical framework, are ideally

110 suited to this task. Recent efforts to simulate and predict the locations of historic and future

111 Ebola virus and Lassa fever outbreaks in West Africa (from environmental, host and

112 epidemiological data using 'environmental-mechanistic models') demonstrate the potential of

113 systems models in forecasting emerging disease risk, but to date these are relatively limited in

114 scope, focusing on single pathogens and omitting aspects of within-host pathogen dynamics ${ }^{28,29}$. 
115 We show the relevance of predictive systems ecology models to epidemiology by

116 explaining how they could be developed and applied to forecast and ultimately improve our

117 understanding of pathogen community ecology and how this translates to emerging disease risk.

118 From these models — which we term 'General Episystem Models' (GEpMs) — the dynamics of

119 functionally similar pathogens would emerge from the cumulative responses of parasites, their

120 hosts and vectors to environmental inputs, rooted in ecological and evolutionary theory. To

121 ground these efforts in real-world episystems, we propose model refinement and validation as

122 part of a global experimental network representing replicates across a common set of

123 anthropogenic environmental drivers for disease emergence (e.g., habitat fragmentation,

124 agricultural intensification, pollution, urbanization) in terrestrial and marine environments.

125 Experimental and observational data could be used to develop and validate standardized

126 approximations for describing broad-scale levels of host and parasite organization (genetic,

127 individual, population, community) and their interactions under different environmental

128 conditions across spatial and biological scales.

\section{$130 \quad$ System structure}

131 Host, Pathogen and Vector Population Dynamics. Where possible, and in common with

132 general ecosystem models, fundamental concepts and processes derived from ecological and 133 epidemiological theory (many of which already exist and are backed up by data) should be used

134 as general baselines with which to model host, parasite and vector population dynamics ${ }^{24}$. The 135 complexity of microbial ecology and evolution, its relative infancy as a field of study, and our 136 lack of knowledge on parasite diversity ${ }^{30}$, mean that uncertainty will pose a major challenge in 137 incorporating pathogen community ecology into predictive systems ecology models. While 
138 GEpMs should be no more complex than is necessary to realistically represent episystems,

139 sufficient information on the biological organization of parasites, their hosts and vectors, and the

140 interactions and feedback between this triad and their abiotic and biotic environments, is required

141 for emergent behaviors of pathogen communities and the risk that they pose to humans to be

142 considered reliable. Applying simplifying assumptions as a means of reducing complexity in

143 these models will therefore be central to achieving a balance between predictive accuracy, and

144 methodological and computational feasibility (Figure 2).

145 A simple but effective form of dimension reduction commonly used in community

146 ecology, and favored for predictive systems ecology models, involves grouping organisms that

147 share life history traits. These similarities dictate that they interact with one another and their

148 environment in a similar manner, so that they are considered identically for modelling purposes.

149 For example, by grouping organisms into functional groups, the Madingley Model has been able

150 to capture global patterns in broad ecosystem structure with a reasonable degree of accuracy ${ }^{26}$.

151 Similarly, trait-based grouping of parasites has been identified as an approach that would

152 contextually simplify modelling of complex within- and between-host pathogen dynamics, and

153 being more directly relevant to ecosystem function, provide greater deterministic and predictive

154 power than taxonomic groupings $9^{9,31,32}$. Representing parasites, hosts and vectors as cohorts that

155 share common resource mechanisms and functional traits (e.g., immune evasion strategies for

156 pathogens, and reproductive and feeding preferences for pathogens, commensal organisms, hosts

157 and vectors), could therefore provide much-needed simplification to overcome data paucity and

158 the logistical challenges of trying to model all individuals in large and complex episystems (Box

1591 , Table 1$)^{26,33}$. By simplifying and compartmentalizing GEpMs in this way, these models would

160 not be able to make predictions about the behavior or emergence of specific pathogens. Rather, 
161 they would possess the predictive power to model how the relative abundance of functionally

162 related groups of pathogens (e.g., reverse-transcribing RNA viruses, extracellular drug-resistant

163 bacteria, intracellular apicomplexans) changes across space and time, while reproducing the

164 cross-scale biological processes that are responsible for this variation (Table 1).

165 Since ecosystem structure and stability is predominantly governed by consumer-resource

166 interactions between species - extending, for example, from cellular invasion of viruses within

167 bats, to the impact of bats on arthropod herbivory of the tropical rainforests that they inhabit ${ }^{34}-$

168 identifying generalizations for these interactions ("food webs") will greatly simplify mechanistic

169 models of the ecological processes that link cohorts of parasites, their hosts, vectors and the

170 environment. Lafferty et al. ${ }^{35}$ demonstrated how classical models of food web structure

171 (including predator-prey, pathogen, autotroph, decomposer and scavenger models) could be used

172 to generate a general consumer-resource model, capturing all forms of species interaction and

173 revealing new insights into the commonalities of different consumer-resource interactions.

174 Recent studies suggest that complex microbial community dynamics can also be predicted by a

175 relatively simple set of rules expressed as species functional traits and metabolic properties of the

176 environment (such as nutrient availability) ${ }^{36,37}$.

177 Because interactions between parasites, hosts, vectors and the environment occur across

178 and between a multitude of microscopic and macroscopic scales, course-grained statistical laws

179 such as allometric scaling rules will also be crucial to identify commonalities that can be used to

180 resolve the underlying interactions between parasite, host and vector communities at a

181 computationally feasible resolution ${ }^{38,39}$. Body mass scaling laws are widely used in ecology, and

182 represent simple predictors of metabolism, abundance, growth and mortality across taxa ${ }^{39}$.

183 Recent work has explored these four scaling laws across all eukaryotes, and found that a scaling 
184 regime based on the ontogenic and reproductive growth of individuals holds consistently across

185 all species, and could therefore be considered a general basis for the assembly of biological

186 communities $^{39}$. Unsurprisingly, scaling rules also apply to microorganisms - a 'dominance'

187 scaling law (representing the number of individuals belonging to the most abundant species in a

188 defined space) predicts microbial diversity from individual plants and animals to the entire

189 ocean's sediment ${ }^{40}$, and log-log scaling rules link gut microbial diversity and animal mass across

190 mammals and birds ${ }^{41}$. With next-generation deep sequencing data being generated at an

191 exponential rate, further unifying principles for biological scaling across eukaryotes and

192 prokaryotes are likely to emerge. Recent work shows that by incorporating allometric scaling of

193 hosts (and other correlative biological relationships) into mechanistic disease transmission, the

194 influence of changes in host communities (such as biodiversity) on pathogen dynamics can be

195 predicted - causal relationships that are difficult to measure directly ${ }^{42,43}$. Collaboration between

196 landscape ecologists, mathematical epidemiologists, immunologists, parasitologists, and disease

197 ecologists who are advancing our understanding of pathogen community ecology, will be

198 required to extend scaling rules to consumer resource models that describe host-pathogen

199 dynamics in multi-agent, multi-host systems across local and regional scales ${ }^{43-45}$.

201 Evolution. GEpMs should also incorporate evolutionary change into parasite and vector

202 population dynamics, as rapid generation times that vary widely between microorganisms

203 (bacteria, viruses fungi), macroparasites and vectors are likely to outpace the duration of model

204 projections. In the simplest terms, parasites could be grouped by evolutionary traits that take into

205 account rates of recombination - for example as clonal or non-clonal organisms ${ }^{46}$ (Box 1, Table

206 1). At a finer resolution, Gorter et al. ${ }^{47}$ propose a general framework to predict the effects of 
207 evolutionary changes on microbial communities, and develop a cellular automaton model for the

208 positive or negative fitness effects of mutations on the composition of a simple, spatially

209 structured microbial community. Others have developed simulation models for the effects of

210 individual-level microbe fitness and host selection on microbiome diversity and the composition

211 of beneficial, commensal, and pathogenic microorganisms ${ }^{48,49}$. How mutualistic or antagonistic

212 interspecific interactions that are conferred by mutation scale to more complex microbial

213 communities is an area of great uncertainty, but there is evidence to suggest that the general form

214 of such interactions at the community level is responsible for shaping microbial assemblages ${ }^{50-}$

$215 \quad 52$. Carefully controlled experimental studies that improve our understanding of how specific

216 traits (gained through mutation or recombination and that are thought to drive the interaction

217 between species) impact fitness, are required to refine these models so that their predictive power

218 can be tested against real-world parasite and vector communities ${ }^{47}$ (Figure 2).

219 Stochastic evolutionary processes (i.e., random genetic variation of pathogens such as

220 genetic drift) will be particularly difficult to model mechanistically and might be best

221 approached using correlative models that generate simple statistical relationships (such as power

$222 \operatorname{laws}^{53}$ ) between patterns of genetic variation within parasite assemblages, community structure

223 and the environment. Recent studies that have successfully predicted evolutionary processes in

224 microbial communities using knowledge of community architecture and environmental

225 conditions provide evidence that microbial community structure can be forecast without

226 requiring a detailed mechanistic understanding of evolutionary processes ${ }^{54,55}$. The increasingly

227 large data sets provided by next-generation, high-throughput sequencing provide a rich resource

228 that can be mined for biologically significant relationships that link pathogen genetics and

229 ecology using machine learning approaches ${ }^{56}$. Parameters derived from correlative models can 
230 then be used to simplify, and parameterize, semi-mechanistic models for parasite evolution and

231 fitness described above ${ }^{54}$ (Figure 2).

232

\section{Parameterizing GEpMs with data}

234 Once a prototype GEpM has been defined from existing knowledge, a large amount of

235 data would be required to refine and validate the system's structure. Because of the extensive

236 scales at which episystems operate, data gathering efforts - both experimental and observational

237 - would need to be undertaken as part of an ambitious cooperative approach that takes place

238 across spatial and temporal scales relevant to the processes being modeled (Figure 2). For such

239 an effort to be practical and cost-effective, experimental design would need to be an iterative

240 process, in which the model is used to highlight data gaps and develop hypotheses, which in turn

241 inform study design and generate results which are utilized to further simplify and constrain the

242 GEpM (Figure 2) $)^{57,58}$. By closely mimicking specific microbiological processes of interest,

243 single-site experimental trials conducted in animal models provide a practical and targeted way

244 of studying the fundamental dynamics (e.g., competition, mutualism, evolution) of parasite

245 communities within the host environment, and identifying feedback loops between parasite

246 communities and their hosts (e.g., via the immune system). Under carefully controlled field

247 conditions, animal models would also be appropriate for studying the mechanisms by which

248 specific abiotic drivers impacting hosts (such as nutritional and psychological stress) and host

249 population dynamics influence the accumulation and turnover of parasite communities.

250 For GEpMs to be parameterized with simplifying assumptions that can account for how

251 environmental inputs (such as land-use and climate) structure parasite, host and vector

252 populations, observational and experimental field data will need to be collected under 'real- 
253 world' conditions. In the first instance, incorporating parasite communities into well-established,

254 long-term studies of intact ecosystems would be an excellent way to test how baseline parasite

255 community dynamics scale across relatively stable ecosystems. For example, sites such as

256 Yellowstone National Park where long-term studies have been conducted on elk, bison, wolves

257 and bears and their interactions within the park provide opportunities to compare the parasitic

258 fauna of predators and prey, seasonal variation in these, and also their interactions with well-

259 studied pathogens such as Brucella spp. in bison and elk and scabies and canine distemper in

260 wolves ${ }^{59,60}$. The diets of grizzly and black bears have been well characterized, as they have for

261 most species in the park, so temporal studies could be applied to examine how life history traits

262 like annual hibernation impact mammalian microbiomes ${ }^{61,62}$. Studies in Yellowstone could be

263 expanded to include data from the Yellowstone to Yukon Conservation Initiative (Y2Y) that has

264 set up experimental sites along a vast longitudinal gradient ${ }^{63}$. This would allow examination of

265 how parasite communities change along a climate gradient that spans multiple ecosystems.

266 The effects of anthropogenic environmental change, which manifests on pathogen

267 community ecology at both fine and broad spatial scales, would need to be studied

268 experimentally and by observation under differing levels of anthropogenic stress. Consider a

269 pastoral grassland system for example. Here, controlled experimental trials in grasslands can

270 provide insight into how local-scale forces (such as agricultural practices) shape host and

271 parasite populations and their interactions with the environment within and between plots ${ }^{64,65}$.

272 Upscaling to landscapes, where the effects of environmental filtering and dispersal on host and

273 vector populations are greatest, observational studies conducted using remote monitoring devices

274 along gradients of human activity (such as the 'Biome Health Project'

275 https://www.biomehealthproject.com/) can be used to estimate how anthropogenic environmental 
276 change impacts the spatial distribution of host and vector populations (e.g., ungulate wildlife,

277 livestock, mosquitos, ticks ${ }^{66}$. When paired with metagenomic and metatranscriptomic

278 sequencing, associations between hosts and their environment can be related to pathogens and

279 their functional roles within parasite communities, through blood-meal or gut content analysis ${ }^{67}$.

280 Collecting these 'real-world' observations over time will be especially important to elucidate

281 evolutionary processes, and perturbations that can disrupt competition between parasites, leading

282 to pathogen colonization ${ }^{51,68,69}$.

283 GEpMs need not be restricted to terrestrial settings, as a similar theory and data gathering

284 approach could be used to develop them for aquatic systems, where the risk posed by infectious

285 diseases is high (such as coastal shorelines). However, in contrast to terrestrial systems, GEpMs

286 would need to be refined to account for differences in aquatic systems that impact the dispersal

287 of pathogens ${ }^{70}$. Experimental trials that focus on aquaculture species could elucidate the

288 dynamics between parasite and host communities, while observational studies conducted at a

289 broader scale could determine the mechanisms that cause certain aquatic habitats, such as

290 marshes $^{71}$ and seagrasses ${ }^{72}$, to remove and potentially destroy human pathogens that invade these

291 habitats. In both terrestrial and aquatic systems, sentinel interfaces deemed important for inter-

292 species disease transmission and zoonotic pathogen spillover would make particularly useful

293 study sites where the experimental approaches outlined above could be used to link patterns of

294 parasite diversity to host and vector population dynamics, and the environment.

295

\section{System dynamics and spillover risk}

297 Once built, a GEpM would simulate how functional groups of pathogens behave under varying

298 environmental and anthropological inputs (e.g., spatially explicit data on climate change, habitat, 
socioeconomics and human distribution), generating results that can be used to evaluate human

300 disease risk across land or seascapes. To achieve this, system structure - comprising cohorts of

301 parasites, their hosts and vectors, each defined by functional traits - would be modelled within

302 grid cells that represent a layer of spatially heterogeneous environmental and anthropological

303 conditions across the land or seascape under consideration ${ }^{26}$ (Box 1, Figure 3). In line with

304 existing general ecosystem models, it wouldn't be unreasonable to expect a process-based GEpM

305 to be capable of simulating episystem dynamics within any ecosystem and at any level of spatial

306 resolution. Properties of pathogen communities (e.g., the relative abundance and biomass of

307 different functional groups) would manifest within each grid cell over consecutive model

308 iterations, emerging from macro-scale processes at the level of individual host and vector

309 cohorts, and in accordance with their responses to environmental and anthropogenic conditions

310 within that grid cell (Figure 3). Comparison of pathogen functional group abundance (and host,

311 and vector abundance and distribution) with empirical data collected within sentinel land and

312 seascapes, would enable validation of the model's results under different environmental

313 scenarios.

314 Incorporating human behavior into GEpMs will be critical to account for the impacts of

315 human activities on pathogen community ecology and generate meaningful estimates of human

316 disease risk. With the exception of administering medical treatments to livestock, we would

317 expect anthropological effects to manifest indirectly on parasite communities through changes in

318 the distribution and composition of host and vector populations resulting from the top-down

319 impacts of climate change, human-mediated introduction of invasive species, land-use change

320 and fragmentation, and variation in livestock-keeping or aquaculture practices. As such, rather

321 than including humans and their activities as agents within the model, GEpMs could follow 
322 general ecosystem models in accounting for human impacts as exogenous factors, incorporated

323 into climatic, land-use, socioeconomic or human demographic layers that are inputs for the

$324 \operatorname{model}^{26}$. For example, a discrete harvesting parameter based upon socioeconomic data could be

325 used to constrain the growth of livestock cohorts with the model. Socioeconomic determinants of

326 livestock keeping are relatively well understood, and models pairing social, economic and

327 ecological systems show that the impacts of humans on the environment and vice-versa can be

328 modelled in a predictive fashion ${ }^{73,74}$.

329 To estimate human spillover risk, predictions for the abundance and distribution of

330 pathogen functional groups made by GEpMs would need to be expressed in terms of human risk.

331 The risk of disease outbreaks in people can be quantitatively expressed by the following

332 equation: Risk = Hazard $\mathrm{x}$ (Vulnerability $\mathrm{x}$ Exposure), where hazard is the availability of

333 pathogens to infect a human at any given time and space, exposure is people's contact with these

334 pathogens, and vulnerability is the likelihood of infection occurring upon contact ${ }^{75}$. General

335 mathematical expressions that use this framework to measure animal-to-human spillover risk

336 have been proposed ${ }^{4,76}$, and in generating estimates of abundance for pathogen cohorts, GEpMs

337 could be used to predict hazard for groups recognized as emergent threats (such as negative-

338 strand RNA viruses, or drug-resistant bacteria) within these models (Figure 3; Box 1).

340 Control and design

341 We think that GEpMs could radically improve our understanding of epidemiological processes

342 occurring in human-modified landscapes, directing surveillance and control efforts for emerging

343 diseases, and ultimately identifying the stability of parasite communities within landscapes.

344 Since forecasting of disease emergence is primarily informed by phenomenological studies ${ }^{77}$, 
345 GEpMs could ensure that health policy decisions are guided by an understanding of how

346 epidemiological systems actually function. For example, applied to ecological systems under

347 anthropogenic stress (we use the examples of a grassland ecosystem in Figure 3 and coastal

348 ecosystems in Suppl. Figure 1), GEpMs could be used to create dynamic risk maps for priority

349 groups of pathogens (e.g., negative-strand RNA viruses which include zoonotic viruses

350 responsible for Ebola, hantaviruses, influenza, and rabies), and forecast how these might change

351 in response to climate change, land-use change, population and socioeconomic trends. Because

352 pathogen dynamics would emerge from spatially explicit environmental and socioeconomic data,

353 computers of the future could run these models at broad spatial scales to provide real-time

354 forecasting for priority groups of pathogens.

355 Once armed with a more detailed quantitative and mechanistic understanding of the role

356 of parasites in natural ecosystems, a key question remains how progress can be made towards

357 preventing and controlling outbreaks of infectious agents, or breakdowns in ecosystem services.

358 The best way to confront this might be to 'reverse engineer' these problems. For example, we

359 know that vital ecosystem services such as the cleansing of air and water are driven by a

360 diversity of species within the ecosystem. If these ecosystem functions could be characterized as

361 outputs from general ecosystem or episystem models, it would be possible to examine the ways

362 in which their relative production declines as the abundance and diversity of species that drive

363 the pathways changes (sensu Dobson et al. ${ }^{78}$ ). Applying these principles to emerging infectious

364 diseases, where the primary drivers of animal-to-human spillover are known to be the wildlife

365 trade, and destruction and fragmentation of tropical forests, GEpMs could be used to identify

366 species that carry significant burdens of pathogens with characteristics that would make their

367 appearance in the wildlife trade particularly problematic (low specificity, unusual range of 
368 hosts). What would this then tell us about minimizing species loss and reductions in abundance

369 in ways that minimize loss of ecosystem function and reduce risk of human exposure to

370 emerging pathogens? Armed with knowledge of the ecological mechanisms that systematically

371 control the state of host and pathogen communities, novel targets for mitigating spillover risk

372 could be identified and tested ${ }^{9}-$ such as creating spatial buffers between hosts, managing habitat

373 to control host and vector populations ${ }^{79}$, or encouraging changes in livestock-keeping practices

374 and other behavioral risk factors for disease emergence ${ }^{80}$. In this way, strategies to modify

375 epidemiological processes and thereby disrupt pathogen spillover, could be designed on the basis

376 of 'in-silica' simulation.

377 The considerable challenges associated with developing these models, and their

378 limitations, should be recognized. As is the case for general ecosystem models, acquiring

379 sufficient data to parameterize and validate GEpMs represents a significant obstacle to their

380 development. We therefore suggest that initial efforts focus on developing GEpMs for areas

381 where long-term studies of free-living species are ongoing, and where concerns are increasingly

382 expressed that pathogens play a crucial but only partially understood role in structuring

383 communities of hosts. For example, longstanding ecological monitoring projects in ecosystems

384 such as Yellowstone ${ }^{81,82}$, The Serengeti ${ }^{83}$, Gorongosa ${ }^{84}$ and the Galápagos National Parks, where

385 rich historical datasets of pathogen prevalence exist from different trophic guilds of hosts, would

386 provide valuable resources with which to begin parameterizing and validating GEpMs ${ }^{85-87}$. To

387 scale predictions beyond well-characterized sentinel landscapes and achieve the impact we

388 envisage relating to predicting emerging disease risk, a coordinated global effort will be

389 required. Although daunting, the challenge of conducting and connecting studies that scale from

390 individual hosts, to host populations in experimental plots and across landscapes, could be met 
391 by a distributed experimental network - a collaborative effort between scientists, consisting of

392 multifactorial studies replicated across many sites, and conducted using standardized protocols

393 that enable comparison and sharing of data ${ }^{88}$. This form of collaboration across sites is not

394 without precedent in ecology - for example the US National Science Foundation's National

395 Ecological Observatory Network (NEON) — which is now collecting data on host and parasite

396 communities) ${ }^{89,90}$ —and the Smithsonian's Forest Global Earth Observatory (ForestGEO) ${ }^{91}$ and

397 Marine Global Earth Observatory (MarineGEO) networks, apply rigorous, standardized data

398 collection protocols across sites to monitor long-term ecological change. The availability of

399 high-resolution geospatial observations, coupled with rapid advances in autonomous biosensing

400 technology, promise the ability to collect large quantities of biological data across spatial and

401 ecological scales, and at relatively low cost.

402 Although a sizeable initial grant would be required to establish such a network on an

403 international scale, the necessary expansion would be constrained by hypotheses generated by

404 the model, and costs could be offset through the contribution of these efforts towards mitigation

405 of disease emergence and future pandemics ${ }^{92}$. An experimental network based on voluntary

406 participation, in which contributors benefit from the results of the model by submitting their data

407 to help improve it, would reduce costs and extend its reach into under-resourced areas, paying

408 dividends over the long-term. Finally, to scale predictions of spillover risk beyond well-

409 characterized sentinel landscapes, detailed global inventories of hosts, vectors and their parasites

410 will be required. Large-scale data-gathering programs already exist for phenotypic and genetic

411 diversity of vertebrates, vectors and their pathogens (e.g. PanTHERIA, ViPR (Virus Pathogen

412 resource), NCBI GenBank, VectorBase, Barcode of Life Database (BOLD)) and proposed 
413 initiatives such as the Global Virome Project ${ }^{93}$ and a Global Parasite Project ${ }^{30}$ will be central to 414 these global efforts.

415 Progress in linking complex parasite-host-environment systems with elegant

416 mathematical expressions would represent huge advances in the fields of disease ecology, and

417 success should therefore not be assumed. The computational power required to simulate complex

418 systems is a major hurdle. Nevertheless, the development of global general ecosystem models

419 has proven to be achievable by reducing dimensionality (grouping organisms into functional

420 groups, and cohorts within functional groups $)^{26}$. Because GEpMs would necessarily simplify

421 episystems into trait-based groups of pathogens, they will not possess the predictive power to

422 model the behavior of specific pathogens, or determine exactly where and when new pathogens

423 will emerge. For this reason, where the goal is to inform management of the risk associated with

424 specific diseases, we recommend that GEpMs are coupled with more traditional epidemiological

425 models/approaches. By unlocking broader principles that underlie epidemiological processes

426 (sensu Lafferty et al. ${ }^{35}$ ), GEpMs could lead to breakthroughs in the design of more detailed,

427 accurate statistical or agent-based models of specific diseases, while identifying areas that

428 require further investigation.

429 In the midst of a global pandemic of wildlife origin, the need for models that consider the

430 full ecological and anthropological contexts of disease transmission is clear. By challenging

431 scientists to reconstruct epidemiological processes from the bottom-up and on the basis of

432 ecological principles, systems models could form a new frontier in epidemiology, uncovering

433 new processes and ultimately improving our understanding of disease emergence, and ability to

434 target surveillance activities and interventions at a global scale. The potential benefits to 
435 understanding health across species, communities and ecosystems across the planet are

436 enormous.

\section{$438 \quad$ References cited}

440 1. Allen, T. et al. Global hotspots and correlates of emerging zoonotic diseases. Nat.

441 Commun. 8, (2017).

442 2. Gibb, R. et al. Zoonotic host diversity increases in human-dominated ecosystems. Nature $443 \mathbf{5 8 4}, 398-402(2020)$.

444 3. Hassell, J. M., Begon, M., Ward, M. J. \& Fèvre, E. M. Urbanization and Disease

445 Emergence: Dynamics at the Wildlife-Livestock-Human Interface. Trends Ecol. Evol. 32, 446 55-67 (2017).

447 4. Plowright, R. K. et al. Pathways to zoonotic spillover. Nat. Rev. Microbiol. 15, 502-510 448 (2017).

449 5. Holmes, E. C., Rambaut, A. \& Andersen, K. G. Pandemics: Spend on surveillance, not $450 \quad$ prediction comment. Nature 558, 180-182 (2018).

451 6. Tabachnick, W. J. Challenges in predicting climate and environmental effects on vector452 borne disease episystems in a changing world. J. Exp. Biol. 213, 946-954 (2010).

453 7. Franklinos, L. H. V., Jones, K. E., Redding, D. W. \& Abubakar, I. The effect of global 454 change on mosquito-borne disease. The Lancet Inf. Dis. 19, e302-e312 (2019).

455 8. Seabloom, E. W. et al. The community ecology of pathogens: Coinfection, coexistence 456 and community composition. Ecol. Lett. 18, 401-415 (2015).

457 9. Johnson, P. T. J., De Roode, J. C. \& Fenton, A. Why infectious disease research needs $458 \quad$ community ecology. Science vol. 3491259504 (2015).

459 10. Parker, I. M. et al. Phylogenetic structure and host abundance drive disease pressure in $460 \quad$ communities. Nature 520, 542-544 (2015).

461 11. Telfer, S. et al. Species interactions in a parasite community drive infection risk in a 462 wildlife population. Science 330, 243-6 (2010).

463 12. Dallas, T. A., Laine, A.-L. L. \& Ovaskainen, O. Detecting parasite associations within 464 multi-species host and parasite communities. Proc. R. Soc. B Biol. Sci. 286, 20191109 
(2019).

466 13. Weinstein, S., Titcomb, G., Agwanda, B., Riginos, C. \& Young, H. Parasite responses to large mammal loss in an African savanna. Ecology 98, 1839-1848 (2017).

14. Anderson, R. \& May, R. Infectious diseases of humans: dynamics and control. doi:10.1016/0966-842x(93)90094-8.

15. Keeling, M. J. \& Rohani, P. Modeling infectious diseases in humans and animals. Modeling Infectious Diseases in Humans and Animals (Princeton University Press, 2011). doi:10.1016/s1473-3099(08)70147-6.

16. Buhnerkempe, M. G. et al. Eight challenges in modelling disease ecology in multi-host, multi-agent systems. Epidemics 10, 26-30 (2014).

17. Cross, P. C., Prosser, D. J., Ramey, A. M., Hanks, E. M. \& Pepin, K. M. Confronting models with data: the challenges of estimating disease spillover. Philos. Trans. R. Soc. B Biol. Sci. 374, 20180435 (2019).

18. Johnson, E. E., Escobar, L. E. \& Zambrana-Torrelio, C. An Ecological Framework for Modeling the Geography of Disease Transmission. Trends Ecol. Evol. 34, 655-668 (2019).

19. Warton, D. I. et al. So Many Variables: Joint Modeling in Community Ecology. Trends 482 Ecol. Evol. 30, 766-779 (2015).

20. Carlson, C. J. et al. The global distribution of Bacillus anthracis and associated anthrax risk to humans, livestock and wildlife. Nat. Microbiol. 4, 1337-1343 (2019).

21. Sutherland, W. J. Predicting the ecological consequences of environmental change: A review of the methods. J.Applied Ecol. 43, 599-616 (2006).

22. Getz, W. M. et al. Making ecological models adequate. Ecol. Lett. 21, 153-166 (2018).

23. Carlson, C. J., Chipperfield, J. D., Benito, B. M., Telford, R. J. \& O’Hara, R. B. Species distribution models are inappropriate for COVID-19. Nature Ecology and Evolution, 4 770-771 (2020).

24. Evans, M. R. et al. Predictive systems ecology. Proc. R. Soc. B Biol. Sci. 280, 2013145220131452 (2013).

25. Purves, D. \& Pacala, S. Predictive models of forest dynamics. Science 320,1452-1453 (2008).

26. Harfoot, M. B. J. et al. Emergent Global Patterns of Ecosystem Structure and Function 
from a Mechanistic General Ecosystem Model. PLoS Biol. 12, (2014).

497 27. Lafferty, K. D. et al. Parasites in food webs: The ultimate missing links. Ecol. Lett. 11, 533-546 (2008).

28. Redding, D. W. et al. Impacts of environmental and socio-economic factors on emergence and epidemic potential of Ebola in Africa. Nat. Commun. 10, 1-11 (2019).

29. Redding, D. W., Moses, L. M., Cunningham, A. A., Wood, J. \& Jones, K. E.

30. Carlson, C. J., Dallas, T. A., Alexander, L. W., Phelan, A. L. \& Phillips, A. J. What would it take to describe the global diversity of parasites? Proc. R. Soc. B Biol. Sci. 287, 20201841 (2020).

33. Purves, D. et al. Time to model all life on Earth. Nature 493, 295-297 (2013).

34. Kalka, M. B., Smith, A. R. \& Kalko, E. K. V. Bats limit arthropods and herbivory in a tropical forest. Science vol. 32071 (2008).

35. Lafferty, K. D. et al. A general consumer-resource population model. Science. 349, 854857 (2015).

37. Goldford, J. E. et al. Emergent simplicity in microbial community assembly. Science. 361, 469-474 (2018).

522 38. Hatton, I. A. et al. The predator-prey power law: Biomass scaling across terrestrial and aquatic biomes. Science. 349, 6252 (2015).

524 39. Hatton, I. A., Dobson, A. P., Storch, D., Galbraith, E. D. \& Loreau, M. Linking scaling 525 laws across eukaryotes. Proc. Natl. Acad. Sci. U. S. A. 116, 21616-21622 (2019). 
Acad. Sci. U. S. A. 113, 5970-5975 (2016).

528 41. Godon, J. J., Arulazhagan, P., Steyer, J. P. \& Hamelin, J. Vertebrate bacterial gut diversity: Size also matters. BMC Ecol. 16, 12 (2016).

530 42. Faust, C. L. et al. Null expectations for disease dynamics in shrinking habitat: dilution or amplification? Philos. Trans. R. Soc. B Biol. Sci. 372, 20160173 (2017).

43. De Leo, G. A. \& Dobson, A. P. Allometry and simple epidemic models for microparasites. Nature 379, 720-722 (1996).

44. Strauss, A. T., Shoemaker, L. G., Seabloom, E. W. \& Borer, E. T. Cross-scale dynamics in community and disease ecology: relative timescales shape the community ecology of pathogens. Ecology 100, (2019).

45. Handel, A. \& Rohani, P. Crossing the scale from within-host infection dynamics to between-host transmission fitness: A discussion of current assumptions and knowledge. Philos. Trans. R. Soc. B Biol. Sci. 370, (2015).

46. Tibayrenc, M. \& Ayala, F. J. Reproductive clonality of pathogens: A perspective on pathogenic viruses, bacteria, fungi, and parasitic protozoa. Proc. Natl. Acad. Sci. USA 109, E3305-E3313 (2012).

47. Gorter, F. A., Manhart, M. \& Ackermann, M. Understanding the evolution of interspecies

49. Zeng, Q., Sukumaran, J., Wu, S. \& Rodrigo, A. Neutral Models of Microbiome Evolution. PLOS Comput. Biol. 11, e1004365 (2015).

50. Liautaud, K., van Nes, E. H., Barbier, M., Scheffer, M. \& Loreau, M. Superorganisms or loose collections of species? A unifying theory of community patterns along environmental gradients. Ecol. Lett. 22, 1243-1252 (2019).

51. Coyte, K. Z., Schluter, J. \& Foster, K. R. The ecology of the microbiome: Networks, competition, and stability. Science. 350, 663-666 (2015).

52. Wright, E. S. \& Vetsigian, K. H. Inhibitory interactions promote frequent bistability among competing bacteria. Nat. Commun. 7, 1-7 (2016).

53. Hekstra, D. R. \& Leibler, S. Contingency and statistical laws in replicate microbial closed 
ecosystems. Cell 149, 1164-1173 (2012).

54. Lopatkin, A. J. \& Collins, J. J. Predictive biology: modelling, understanding and harnessing microbial complexity. Nat. Rev. Microbiol. 18, 507-520 (2020).

55. Sanchez, A. \& Gore, J. Feedback between Population and Evolutionary Dynamics Determines the Fate of Social Microbial Populations. PLoS Biol. 11, e1001547 (2013).

56. Wu, F. et al. A unifying framework for interpreting and predicting mutualistic systems. Nat. Commun. 10, 1-10 (2019).

57. Restif, O. et al. Model-guided fieldwork: practical guidelines for multidisciplinary research on wildlife ecological and epidemiological dynamics. Ecol. Lett. 15, 1083-94 (2012).

58. Herzog, S. A., Blaizot, S. \& Hens, N. Mathematical models used to inform study design or surveillance systems in infectious diseases: A systematic review. BMC Infect. Dis. 17, 110 (2017).

59. Cotterill, G. G. et al. Winter feeding of elk in the Greater Yellowstone Ecosystem and its effects on disease dynamics. Philos. Trans. R. Soc. B Biol. Sci. 373, 20170093 (2018).

60. Cross, P. C. et al. Estimating distemper virus dynamics among wolves and grizzly bears using serology and Bayesian state-space models. Ecol. Evol. 8, 8726-8735 (2018).

61. Hopkins, J. B., Ferguson, J. M., Tyers, D. B. \& Kurle, C. M. Selecting the best stable isotope mixing model to estimate grizzly bear diets in the Greater Yellowstone Ecosystem. PLoS One 12, e0174903 (2017).

62. Schwartz, C. C. et al. Body and diet composition of sympatric black and grizzly bears in the Greater Yellowstone Ecosystem. J. Wildl. Manage. 78, 68-78 (2014).

63. Chester, C. C. Yellowstone to Yukon: Transborder conservation across a vast international landscape. Environ. Sci. Policy 49, 75-84 (2015).

64. Young, H. S. et al. Interacting effects of land use and climate on rodent-borne pathogens in central Kenya. Philos. Trans. R. Soc. B Biol. Sci. 372, 20160116 (2017).

65. Sitters, J., Kimuyu, D. M., Young, T. P., Claeys, P. \& Olde Venterink, H. Negative effects of cattle on soil carbon and nutrient pools reversed by megaherbivores. Nat. Sustain. 3, 360-366 (2020).

66. Sethi, S. S., Ewers, R. M., Jones, N. S., Orme, C. D. L. \& Picinali, L. Robust, real-time and autonomous monitoring of ecosystems with an open, low-cost, networked device. 
Methods Ecol. Evol. 9, 2383-2387 (2018).

590 67. Alfano, N., Dayaram, A. \& Tsangaras, K. Non-invasive surveys of mammalian viruses using environmental DNA. bioRxiv Mol. Biol. 2020.03.26.009993 (2020)

68. Coyte, K. Z. \& Rakoff-Nahoum, S. Understanding Competition and Cooperation within the Mammalian Gut Microbiome. Current Biology vol. 29 R538-R544 (2019).

69. Murray, M. H. et al. Gut microbiome shifts with urbanization and potentially facilitates a zoonotic pathogen in a wading bird. PLoS One 15, (2020).

70. McCallum, H. I. et al. Does terrestrial epidemiology apply to marine systems? Trends Ecol. Evol. 19 585-591 (2004).

71. Wu, S., Carvalho, P. N., Müller, J. A., Manoj, V. R. \& Dong, R. Sanitation in constructed wetlands: A review on the removal of human pathogens and fecal indicators. Sci. Total. Environ. 541 8-22 (2016).

73. Janssen, M. A., Walker, B. H., Langridge, J. \& Abel, N. An adaptive agent model for analysing co-evolution of management and policies in a complex rangeland system. Ecol. Modell. 131, 249-268 (2000).

74. Ngonghala, C. N. et al. General ecological models for human subsistence, health and poverty. Nat. Ecol. Evol. 1, 1153-1159 (2017).

75. Hosseini, P. R. et al. Does the impact of biodiversity differ between emerging and endemic pathogens? The need to separate the concepts of hazard and risk. Philos. Trans. R. Soc. B Biol. Sci. 372, 20160129 (2017).

76. Washburne, A. D. et al. Percolation models of pathogen spillover. Philos. Trans. R. Soc. B Biol. Sci. 374, 20180331 (2019).

613 77. Olival, K. J. et al. Host and viral traits predict zoonotic spillover from mammals. Nature $614 \quad$ 546, 646-650 (2017).

615 78. Dobson, A. et al. HABITAT LOSS, TROPHIC COLLAPSE, AND THE DECLINE OF ECOSYSTEM SERVICES. Ecology 87, 1915-1924 (2006).

617 79. Faust, C. L. et al. Pathogen spillover during land conversion. Ecol. Lett. 21, 471-483 618 (2018).

619 80. Sokolow, S. H. et al. Ecological interventions to prevent and manage zoonotic pathogen 
spillover. Philos. Trans. R. Soc. B Biol. Sci. 374, 20180342 (2019).

621 81. Kauffman, M. J. et al. Landscape heterogeneity shapes predation in a newly restored predator-prey system. Ecol. Lett. 10, 690-700 (2007).

623 82. Smith, D. W., Peterson, R. O. \& Houston, D. B. Yellowstone after wolves. BioScience. 53, 330-340 (2003).

83. McNaughton, S. J. Ecology of a Grazing Ecosystem: The Serengeti. Ecol. Monogr. 55, 259-294 (1985).

84. Atkins, J. L. et al. Cascading impacts of large-carnivore extirpation in an African ecosystem. Science. 364, 173-177 (2019).

85. Cross, P. C., Edwards, W. H., Scurlock, B. M., Maichak, E. J. \& Rogerson, J. D. Effects of Management and Climate on Elk Brucellosis in the Greater Yellowstone Ecosystem. Ecol. Appl. 17, 957-964 (2007).

86. Almberg, E. S., Cross, P. C. \& Smith, D. W. Persistence of canine distemper virus in the Greater Yellowstone Ecosystem's carnivore community. Ecol. Appl. 20, 2058-2074 (2010).

87. Holdo, R. M. et al. A Disease-Mediated Trophic Cascade in the Serengeti and its Implications for Ecosystem C. PLoS Biol. 7, e1000210 (2009).

88. Borer, E. T. Ecological Scaling in Time and Space. in Unsolved Problems in Ecology (eds. Dobson, A. P., Holt, R. D. \& Tilman, D.) 3-15 (Princeton University Press, 2020).

90. Springer, Y. P. et al. Tick-, mosquito-, and rodent-borne parasite sampling designs for the National Ecological Observatory Network. Ecosphere 7, (2016).

643 91. Anderson-Teixeira, K. J. et al. CTFS-ForestGEO: A worldwide network monitoring forests in an era of global change. Glob. Chang. Biol. 21, 528-549 (2015).

645 92. Dobson, A. P. et al. Ecology and economics for pandemic prevention. Science 369, 379$646 \quad 381(2020)$.

647 93. Kress, W. J., Mazet, J. A. K. \& Hebert, P. D. N. Opinion: Intercepting pandemics through 648 genomics. Proc. Natl. Acad. Sci. 117, 202009508 (2020).

649 94. Pedersen, A. B. \& Fenton, A. Emphasizing the ecology in parasite community ecology. Trends Ecol. Evol. 22, 133-139 (2007). 
651 95. Plowright, R. K. et al. A call to action: Understanding land use-induced zoonotic spillover to protect environmental, animal, and human health. EcoEvoRxiv (2020) doi:10.32942/OSF.IO/CRU9W.

654 96. Barychka, T., Mace, G. \& Purves, D. The Madingley General Ecosystem Model predicts bushmeat yields, species extinction rates and ecosystem-level impacts of bushmeat harvesting. Cold Spring Harb. Lab. Cold Spring Harb. NY, USA. 2020.03.02.959718 (2020) doi:10.1101/2020.03.02.959718.

97. Durmuş, S. \& Ülgen, K. Comparative interactomics for virus-human protein-protein interactions: DNA viruses versus RNA viruses. FEBS Open Bio 7, 96-107 (2017).

98. Becker, D. J. \& Albery, G. F. Expanding host specificity and pathogen sharing beyond viruses. Mol. Ecol. 29, 3170-3172 (2020).

99. Rittershaus, E. S. C., Baek, S. H. \& Sassetti, C. M. The normalcy of dormancy: Common themes in microbial quiescence. Cell Host Microbe. 13, 643-651 (2013).

100. Schmid-Hempel, P. Immune defence, parasite evasion strategies and their relevance for 'macroscopic phenomena' such as virulence. Philos. Trans. R. Soc. B Biol. Sci. 364, 8598 (2009).

\section{Acknowledgements}

670 J.H., D.Z. \& Y.M.L. were supported by the US Army Medical Research and Development

671 Command under Contract No.W81XWH-21-C-0001 and the Armed Forces Health Surveillance

672 Division - Global Emerging Infections Surveillance branch (AFHSC-GEIS) award

673 P0031_21_WR. The views, opinions and/or findings contained in this report are those of the

674 author(s) and should not be construed as an official Department of the Army position, policy or

675 decision unless so designated by other documentation. T.N. is supported by two grants from the 676 Leverhulme Trust (RPG-2015-073 and RPG-2018-069). 
J.M.H. conceptualized the structure and content of the manuscript and wrote an initial draft.

680 J.M.H., T.N., A.P.D., Y.L., L.V.H.F., D.Z. and K.M.P. expanded upon the ideas contained

681 within this initial draft, and engaged in discussion and editing of the final manuscript.

682

683 Corresponding author

684 Correspondence to James M. Hassell (hasselljm@ @i.edu)

685

686 Box 1: Modeling parasites as cohorts

687 Grouping individuals by their ecological traits is the principal form of dimension reduction used

688 in General Ecosystem Models (GEMs), and an approach that we propose could also be applied

689 when developing GEpMs. In terrestrial GEMs, autotrophs (plants) and heterotrophs

690 (herbivorous, omnivorous, and carnivorous animals) are grouped by nutrition source, mobility,

691 leaf strategy (autotrophs), mobility, reproductive strategy, and thermoregulation mode

692 (heterotrophs). GEpMs would extend GEMs, adding parasites as a second group of heterotrophs

693 that are modelled differently to their hosts (see Harfoot et al. ${ }^{26}$ for a detailed description of how

694 autotrophs and heterotrophs are modelled in GEMs). Drawing on generalized frameworks

695 developed by Pedersen \& Fenton ${ }^{94}$, Lafferty et al. $^{35}$ and Lello \& Hussell ${ }^{32}$ we propose six

696 categorical traits that represent the ecological processes conducted by parasites, and their

697 interactions with hosts (Table 1). Once grouped by these traits, the resource exploitation

698 strategies of individual parasites within each cohort would be modelled using the same

699 mathematical expressions that represent; (i) consumption strategy and impact on host fitness; (ii)

700 immune stimulation and immune evasion (e.g., quiescence); (iii) reproduction; (iv) mortality

701 resulting from the host immune system, or as a result of background mortality processes such as 
702 senescence; and (v) dispersion from their current grid cell to another grid cell (Figure 3). The

703 impact of parasites on host fitness (e.g., through consumer strategies that either reduce host

704 fitness to zero or have a density dependent reduction on the reproductive performance of hosts)

705 would feed back into the modelling of host heterotroph cohorts, and their effects on autotroph

706 biomass.

707

708 Case study: hazard posed by negative-strand RNA viruses in changing terrestrial systems.

709 Human-mediated ecosystem change is considered an important driver of animal-to-human

710 pathogen spillover, but the macro-ecological processes by which this occurs are rarely studied

711 and poorly understood ${ }^{95}$. GEpMs would offer a unique opportunity to simulate the impacts of

712 ecosystem changes (e.g., land use change, harvesting of wild animals) on host populations, and

713 emerging pathogens. Using this as a scenario to demonstrate the potential application of

714 GEpM's, we describe how a prototype model could be used to study the dynamics of negative-

715 strand (NS)-RNA viruses in wild animals, generate predictions of the hazard they pose to

716 humans, and design interventions to protect human health. Following the functional groupings in

717 Table 1, models could target parasites described using the categorical traits 'Pathogen |

718 Intracellular-RNA-reverse transcription | Horizontal-direct | Cellular/Humoral/T-helper cell’. By

719 specifying these classifications, important zoonotic viral families such as orthomyxoviruses,

720 paramyxoviruses and filoviruses would be targeted.

$721 \quad$ Figure 3 depicts how modeling studies conducted across grid cells at different resolutions

722 could assess the GEpM's capacity to simulate ecosystem-scale dynamics across trophic levels

723 from which (NS)-RNA virus properties emerge, and generate high-resolution predictions of the

724 relative abundance/biomass of (NS)-RNA viruses at specific sites undergoing ecosystem 
725 changes. By sourcing environmental input data from closely monitored sites experiencing

726 changes in land use over a defined period, and aligning this to the time steps over which

727 simulations occur, the predicted responses of host and parasite cohorts could be evaluated against

728 empirical data on vegetation, host and parasite abundance. A term that simulates harvesting of

729 certain wild animal host cohorts could then be added to the model to investigate how specific

730 changes in trophic structure influence parasite dynamics ${ }^{96}$. As an emergent property of the

731 GEpM, the relative abundance and biomass of the (NS)-RNA virus cohort could estimate

732 'pathogen pressure' for each grid cell on which the model is run - representing the quantity of

733 (NS)-RNA viruses in wildlife to which humans could be exposed at a given point in space and

734 time. Over multiple grid cells, these predictions would represent the distribution of wild animals

735 carrying these pathogens, and the intensity with which they are infected and shedding them (i.e.,

736 persistence and transmission within wild animal populations). When combined with information

737 on human-wildlife interactions and human susceptibility to infection, this data could be used to

738 predict spillover risk at local, national and global scales. Including livestock hosts would

739 increase the accuracy of these models, and we demonstrate how this could be achieved in Figure

$740 \quad 3$.

741 Furthermore, these models could permit "in-silico" design and testing of interventions

742 aimed at maintaining stable population dynamics of species and their pathogens and mediating

743 human behavior in a way that minimizes the impact of land-use change on biodiversity and

744 human health. For example, a GEpM that describes changes in the predator-prey dynamics of

745 non-human primates in response to fragmentation of tropical forests, and predicts how this

746 impacts their exposure to zoonotic viruses, could be used to forecast the human health risks

747 posed by hunting these species within a given area, and target educational campaigns at 
748 communities who rely on non-human primates as a food source. As new empirical findings

749 emerge, GEpMs could be used to scale and test competing hypotheses for how ecosystem

750 stressors impact host assemblages and the (NS)-RNA viruses they carry, identifying critical

751 processes that require further investigation.

752

753

\begin{tabular}{|c|c|c|c|c|c|c|}
\hline Resource & \multicolumn{3}{|c|}{ Reproductive Strategy } & Metabolism & Immune & Evolution \\
\hline $\begin{array}{l}\text { Consumer } \\
\text { Strategy }\left[{ }^{35}\right]\end{array}$ & Location [97] & Dispersal & $\begin{array}{c}\text { Host } \\
\text { Breadth }\left[{ }^{98}\right]\end{array}$ & $\begin{array}{c}\text { Dormancy/Cellular } \\
\text { Quiescence }\left[{ }^{99}\right]\end{array}$ & $\begin{array}{c}\text { Type of } \\
\text { Immune } \\
\text { Response } \\
{\left[{ }^{94,100]}\right.}\end{array}$ & $\begin{array}{c}\text { Clonality } \\
{\left[{ }^{46}\right]}\end{array}$ \\
\hline Castrator & $\begin{array}{l}\text { Intracellular, } \\
\text { DNA reverse } \\
\text { transcription }\end{array}$ & $\begin{array}{l}\text { Horizontal } \\
\text { - direct }\end{array}$ & $\begin{array}{l}\text { Composite } \\
\text { measure for } \\
\text { each } \\
\text { pathogen }\end{array}$ & No dormant phase & Cellular & Clonal \\
\hline $\begin{array}{l}\text { Macroparasite } \\
\text { Pathogen }\end{array}$ & $\begin{array}{l}\text { Intracellular, } \\
\text { DNA non- } \\
\text { reverse } \\
\text { transcription } \\
\text { Intracellular, } \\
\text { RNA reverse } \\
\text { transcription }\end{array}$ & $\begin{array}{l}\text { Horizontal } \\
\text { - indirect } \\
\text { Vertical }\end{array}$ & $\begin{array}{l}\text { functional } \\
\text { group based } \\
\text { on } \\
\text { databases of } \\
\text { host-parasite } \\
\text { associations. }\end{array}$ & $\begin{array}{l}\text { Can perform } \\
\text { dormancy }\end{array}$ & $\begin{array}{l}\text { Humoral } \\
\text { T-helper cell }\end{array}$ & Not clonal \\
\hline Parasitoid & $\begin{array}{l}\text { Intracellular, } \\
\text { RNA non- } \\
\text { reverse } \\
\text { transcription } \\
\text { Intracellular, } \\
\text { binary fission / } \\
\text { horizontal } \\
\text { gene transfer } \\
\text { Extracellular, } \\
\text { within-host, } \\
\text { asexual } \\
\text { Extracellular, } \\
\text { within-host, } \\
\text { sexual } \\
\text { Extracellular, } \\
\text { environmental, } \\
\text { asexual } \\
\text { Extracellular, } \\
\text { environmental, } \\
\text { sexual }\end{array}$ & & & & & \\
\hline
\end{tabular}


Table 1. Parasite functional groups. To simplify the process of modeling diverse parasite communities, we propose splitting parasites into functionally related groups that represent their consumer strategies, reproductive and metabolic processes, interaction with the host's immune response and evolutionary traits. These classifications represent how parasites $i$ ) use host resources (what they eat and how this impacts host fitness), ii) reproduce (how they reproduce, and the mode and extent of their dissemination to other hosts), iii) respond to stressors (whether they are capable of entering dormancy or not), iv) activate the host immune response (components of the host immune system that are stimulated by each pathogen functional group), and $v$ ) evolve (as differentiated by the levels of genetic recombination that parasites undergo).

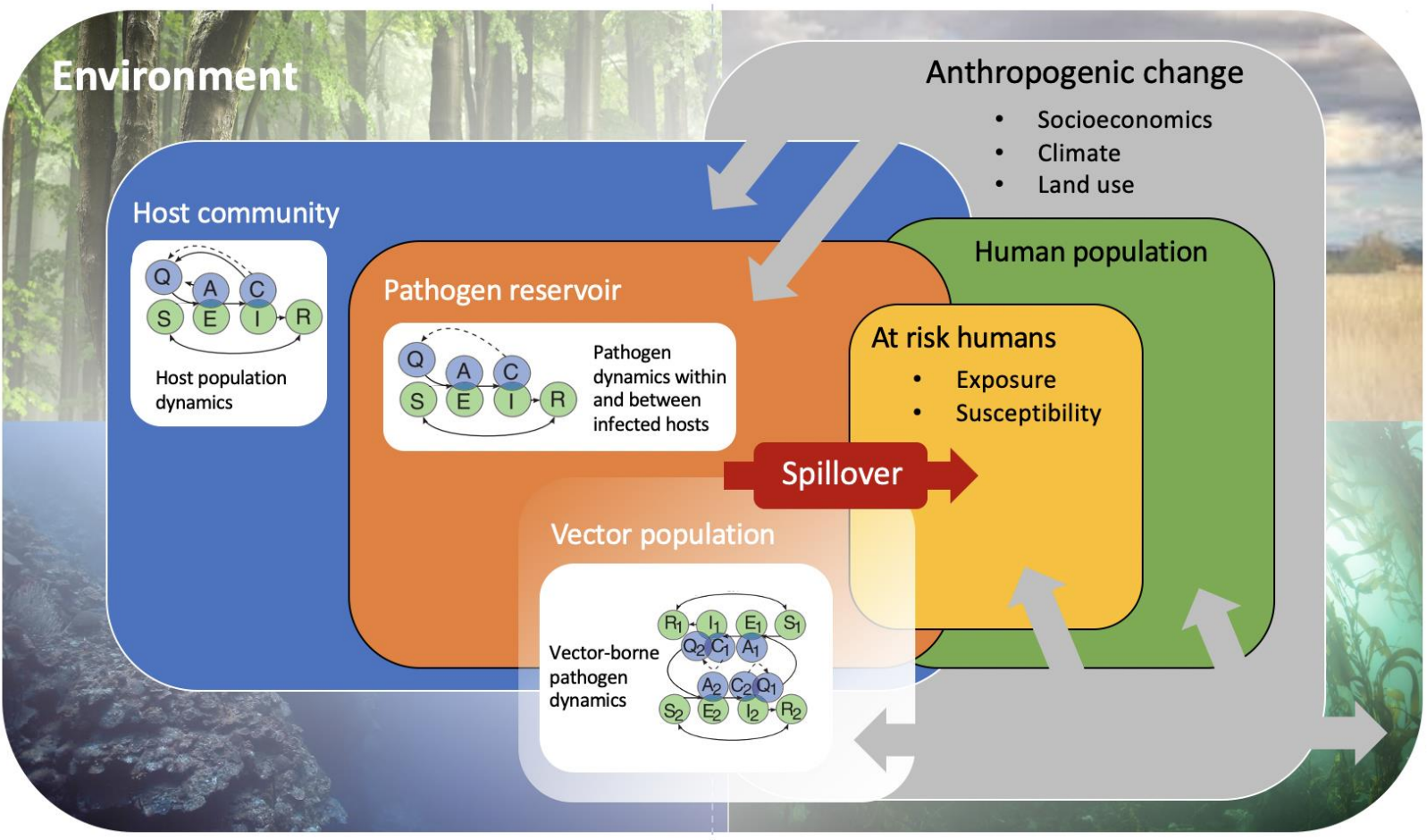

767 Figure 1. Diagrammatic representation of a disease episystem, depicting interactions between pathogens, their hosts and the environment, and the interface for spillover into 
770 kelp forest), and colored boxes nested within this represent host (animal and human), vector and

771 pathogen populations. Anthropogenic factors that drive changes in environment, host and vector

772 populations are depicted in grey, with arrows showing directionality of these effects. White

773 boxes within animal host and vector compartments represent classic consumer-resource models,

774 depicting host-environment, host-pathogen and vector-pathogen interactions (adapted from

775 Lafferty et al. ${ }^{21}$ ). Circles within boxes are state variables for questing (Q), attacking (A), and

776 consuming (C) consumers (blue - predators, or pathogens) and susceptible (S), exposed (E),

777 ingested (I), and resistant (R) resources (green - autotrophs, or hosts). Per Lafferty et al. ${ }^{21}$,

778 arrows represent transitions (of individuals or biomass) among states - a dashed line represents

779 production or conversion (e.g., births), whereas a solid line is a transition from one state to

780 another (implying no change in numbers from one state to the next). Circles numbered " 1 " for

781 the model of vector-borne pathogen dynamics represent processes occurring in the vector, and

782 those numbered " 2 " represent processes occurring in the host.

783 


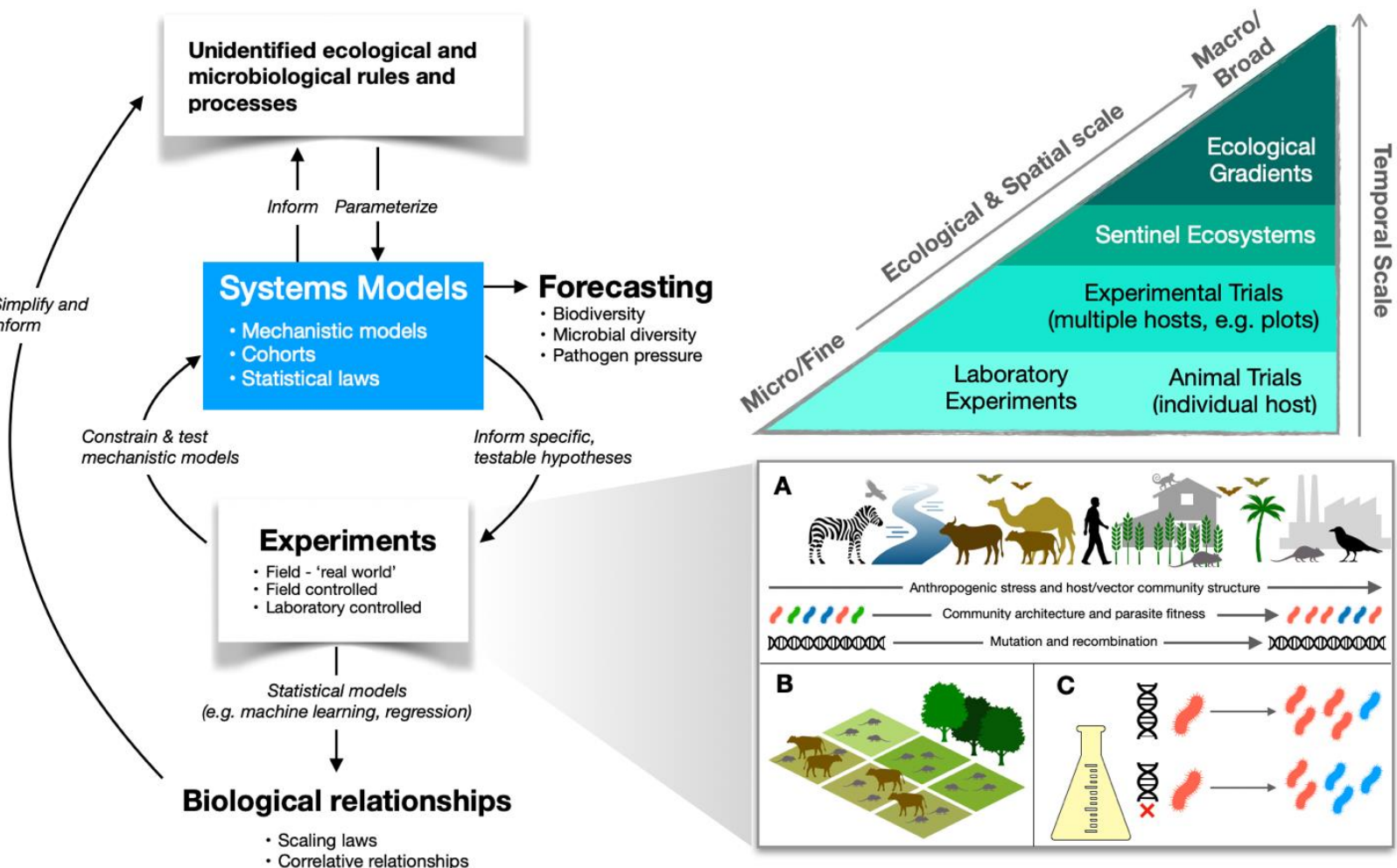

- Scaling laws

- Correlative relationships

Figure 2. Iterative development of an ecosystem model for infectious disease (General

787 Episystems Model - GEpM). Panel 1: Development of an ecosystem model for infectious

788 disease would be an iterative process, in which systems models (collections of interacting models

789 representing the GEpM) are constrained and tested through field and laboratory experiments

790 conducted over varying spatial and temporal scales. In this way, statistical models that explain

791 complex but important relationships could be incorporated into a mechanistic modeling

792 framework, as a means of decreasing complexity while maintaining predictive power. Types of

793 experiment depicted represent a) 'real world' field experiments, where studies investigate

794 species turnover and related evolutionary processes along gradients of anthropogenic stress in

795 ecosystems; b) controlled field trials, where conditions that closely mimic the ecological

796 processes of interest are simulated to improve model accuracy; c) controlled laboratory trials, 
797 where conditions that closely mimic the microbiological (both ecology and evolutionary)

798 processes of interest are simulated to improve model accuracy. To capture the multitude of

799 ecological scales across which parasites interact with one-another and their hosts, and these

800 interactions are filtered by environmental variables, experiments would need to take place across

801 spatial and temporal scales. Together, these experiments also serve to address unanswered

802 questions in ecology and microbiology—as identified during model development—improving

803 predictive capability and simplifying model structure. Panel 2: Initial steps that could be taken

804 towards the development of GEpMs are outlined in this table, along with some of the key

805 challenges facing development of these models.

806

807

808

809

810

811

812

813 


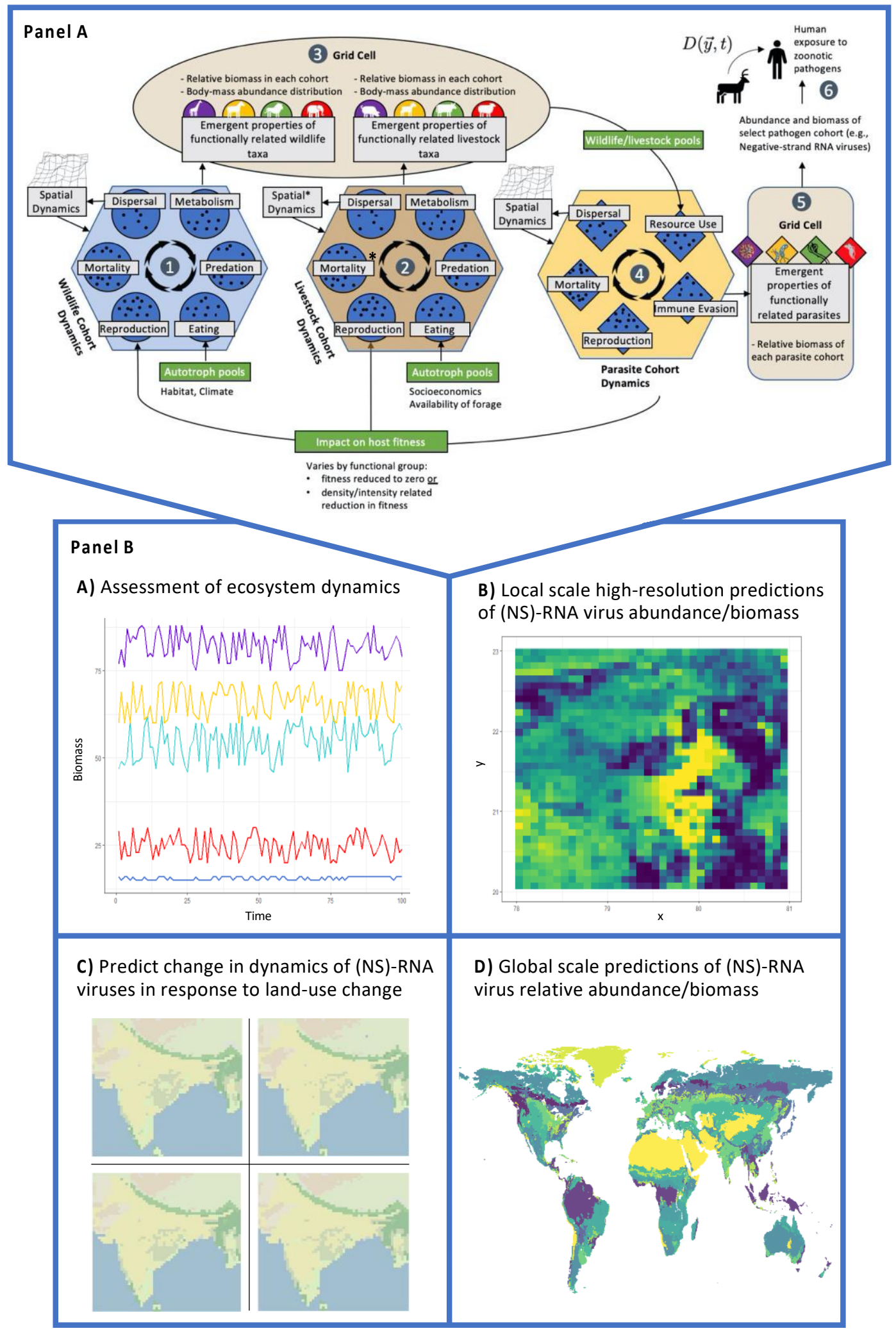


857 are modelled as individuals within cohorts, defined by categorical and quantitative traits.

858 Autotroph biomass (derived from spatially explicit land use per grid cell and climatic variables,

859 economic data and the availability of forage) are used as input data into the wildlife (1) and

860 livestock (2) models. Each grid cell is stocked with initial densities of wildlife, livestock and their

861 parasites, which could be negatively scaled to body masses randomly drawn from a designated

862 range for each cohort $^{26}$. A term that simulates commercial harvesting of livestock could be

863 included in livestock models $(2 *)$. Allometric relationships, combined with spatial models in 1 and

8642 lead to emergent properties of wildlife and livestock cohorts across a grid cell (3). Parasites are

865 also modelled as cohorts of functionally related taxa. Emergent properties of wildlife and livestock

866 cohorts ('host pools') in each grid cell inform allometric relationships between parasites and their

867 hosts, and models which capture transmission between hosts (4). Emergent properties of parasite

868 models feed back to impact host dynamics, and result in measures of parasite community structure

869 that can be projected across grid cells - including the abundance/biomass of pathogen cohorts (5).

870 Mathematical expressions couple changes in host and pathogen dynamics with socioeconomic and

871 behavioral models to predict zoonotic spillover risk (6). Panel B: The GEpM is used to A) make

872 basic assessments of ecosystem dynamics across trophic scales from which (NS)-RNA virus

873 properties emerge, and assess whether these dynamics reach an equilibrium (colors represent

874 different host and parasite cohorts); B) make high-resolution predictions of the relative

875 abundance/biomass of (NS)-RNA viruses at specific sites, where empirical data on vegetation,

876 mammalian and parasite abundance or biomass exist; C) extend these predictions to forecast

877 changes in relative abundance/biomass of (NS)-RNA viruses in response to land-use change or

878 harvesting of certain host cohorts at specific sites, and D) make global, lower-resolution

879 predictions of the relative abundance/biomass of (NS)-RNA viruses 